\title{
An Analytical Approach to the Design of Multiple Mode Rectangular Cavity Waveguide Filters
}

\author{
Ceyhun Kelleci, Student Member, IEEE, and Abdullah Atalar, Fellow, IEEE
}

\begin{abstract}
The multiple mode rectangular cavity structure with square corner cuts is revisited. An attempt to predict the physical dimensions of the cavity for dual mode second-order and triple mode third-order filters is made. Analytic expressions are formed to be used in the design process. The classical triple mode cavity filter structure is altered to give a finite frequency transmission zero either in the lower or upper sideband of the center frequency. The concept is illustrated with example designs. A novel additive manufacturing technique is used to fabricate a selected filter structure. The experimental results are in agreement with the expectations.
\end{abstract}

Index Terms - Additive manufacturing, cavity filter, dispersion, multiple mode cavity, triplet, trisection.

\section{INTRODUCTION}

M ICROWAVE filters have always played an important role in both civilian and military systems and this role becomes more critical with the ever-increasing need for more bandwidth and channels in the spectrum, which is a very scarce resource. On the other hand, more compact lightweight solutions are always welcome. One such filter solution is the multiple mode cavity filter.

The idea of using the degenerate modes within a cavity in the synthesis of a filter was introduced in [1, pp. 673-677] in the late 1940s, whereas a thorough analysis of the idea is carried out in [2]. The necessary coupling between the degenerate modes within the structure is realized by perturbing the geometry of the cavity. The corresponding perturbation elements can be tuning screws as well as square corner cuts [3]. In a more recent work [4], a specific sequence of intermodal couplings in a triple mode rectangular cavity, implemented with similar corner cuts, is proposed and an investigation is made on how a computer-aided design can be realized using the proposed structure.

The main contributions of this paper to the cavity filter design techniques are as follows.

1) Simple closed-form expressions to determine the resonant frequency shifts of the modes of a multiple mode cavity, and the resulting coupling coefficient due to various types of corner cuts are obtained. The resulting expressions are combined with rectangular

Manuscript received August 28, 2016; revised December 14, 2016; accepted February 5, 2017. Date of publication March 10, 2017; date of current version August 4, 2017. The work of A. Atalar was supported by the Turkish Academy of Sciences.

The authors are with the Department of Electrical and Electronics Engineering, Bilkent University, Ankara 06800, Turkey (e-mail: ceyhun@ee.bilkent.edu.tr; aatalar@bilkent.edu.tr).

Color versions of one or more of the figures in this paper are available online at http://ieeexplore.iee.org.

Digital Object Identifier 10.1109/TMTT.2017.2670000 (a)

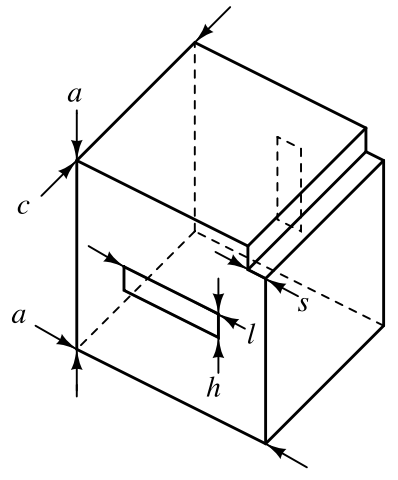

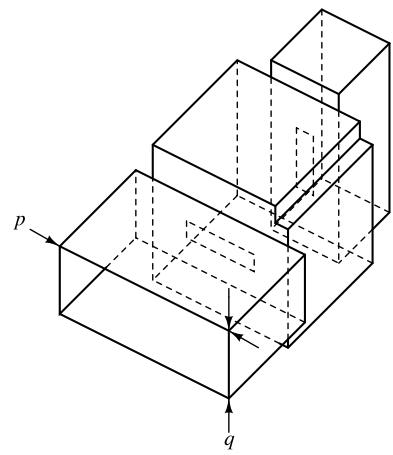

(b)
Fig. 1. Dual mode rectangular cavity filter structure. (a) Cavity. (b) Cavity and waveguide.

aperture-related expressions from the literature to form a complete set of equations that defines the connection between the physical dimensions and the coupling matrix of a second or third-order multiple mode single cavity filter.

2) A filter design algorithm that uses the above-mentioned set of expressions is proposed. The algorithm provides a good starting point in obtaining the optimized dimensions of a single cavity multiple mode filter.

3) An analytical expression determining the dispersive behavior of the rectangular aperture is derived. This expression can be used directly with the coupling matrix to estimate the dispersion caused nonideal response of a filter.

4) The classical triple mode filter structure is altered to form a triplet. The new structure is able to generate either positive or negative cross couplings, hence allowing placement of a finite frequency transmission zero (TZ) either at the upper or lower sideband of the center frequency.

5) The additive manufacturing technology is combined with multiple mode cavity technique for the first time. The combination of the two techniques results in very lightweight filters. An example design illustrating the concept is realized in $\mathrm{Ka}$-band.

\section{Multiple Mode Cavity Filter}

The dual and triple mode cavity filter structures can be seen in Figs. 1 and 2. Both are composed of a single cavity with square corner cuts to generate the intermodal couplings and rectangular apertures to realize the source/load-to-resonator couplings. 


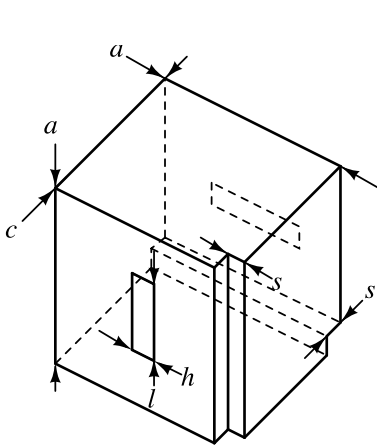

(a)

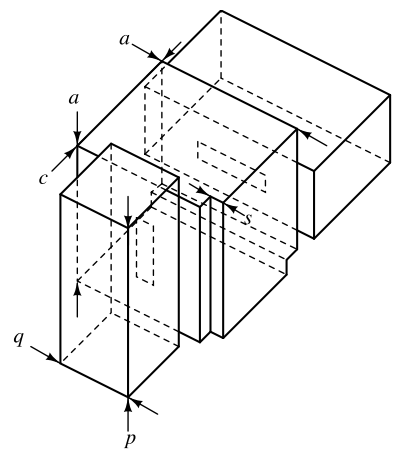

(b)
Fig. 2. Triple mode rectangular cavity filter structure. (a) Cavity. (b) Cavity and waveguide.

$4 \times 4$ coupling matrix corresponding to a Chebyshev secondorder filter is in the following form:

$$
M=\left[M_{i j}\right]=\begin{gathered}
S \\
R_{1} \\
R_{2} \\
L
\end{gathered}\left[\begin{array}{cccc}
S & R_{1} & R_{2} & L \\
0 & c_{S 1} & 0 & 0 \\
c_{S 1} & 0 & c_{12} & 0 \\
0 & c_{12} & 0 & c_{2 L} \\
0 & 0 & c_{2 L} & 0
\end{array}\right]
$$

The only nonzero, thus to be defined, parameters of the coupling matrix are the source to the first resonator coupling $c_{S 1}$, the inter-resonator coupling $c_{12}$, and the load to the last resonator coupling $c_{2 L}$. Due to symmetry, $c_{S 1}=c_{2 L}$.

For a third-order Chebyshev filter, the only nonzero elements of $5 \times 5$ coupling matrix are $M_{12}=M_{21}=M_{45}=$ $M_{54}=c_{S 1}$ and $M_{23}=M_{32}=M_{34}=M_{43}=c_{12}$ with again only two independent parameters.

\section{INTERMODAL COUPLING}

\section{A. Square Corner Cut}

Atia et al. [3] proposed the idea of using square corner cuts instead of tuning screws to couple the degenerate modes of a square cross section waveguide/cavity. The same structure is also used for triple mode cavities [4].

A square cross-sectioned cavity has dual symmetry, hence, it has two degenerate modes. In the presence of a square corner cut, these modes are no longer degenerate and can be interpreted to be coupled to each other. The physical intermodal coupling coefficient, $k_{c}$, can be related to the resonant frequencies, $f_{1}$ and $f_{2}$, of the coupled modes with [3]

$$
k_{c}=\frac{f_{1}^{2}-f_{2}^{2}}{f_{1}^{2}+f_{2}^{2}}
$$

The presence of the square corner cut in a waveguide forces the fields to be diagonal [1, p. 675], [5]. The rectangular cavity field expressions of [6, pp. 155-157] are modified with the diagonal mode approach and are given as

$$
\left\{\begin{array}{l}
\boldsymbol{E}_{\{\mathbf{1 , 2 \}} t}=-\frac{\pi}{a} \sin \left(\frac{\pi z}{c}\right)\left[\sin \left(\frac{\pi y}{a}\right) \hat{\boldsymbol{x}} \pm \sin \left(\frac{\pi x}{a}\right) \hat{\boldsymbol{y}}\right] \\
\boldsymbol{H}_{\{\mathbf{1 , 2}\} t}=j \frac{\pi^{2}}{k_{0} a c} \sqrt{\frac{\epsilon_{0}}{\mu_{0}}} \cos \left(\frac{\pi z}{c}\right)\left[\sin \left(\frac{\pi y}{a}\right) \hat{\boldsymbol{x}} \pm \sin \left(\frac{\pi x}{a}\right) \hat{\boldsymbol{y}}\right] \\
\boldsymbol{H}_{\{\mathbf{1 , 2}\} z}=-j \frac{\pi^{2}}{k_{0} a^{2}} \sqrt{\frac{\epsilon_{0}}{\mu_{0}}} \sin \left(\frac{\pi z}{c}\right)\left[\cos \left(\frac{\pi x}{a}\right) \pm \cos \left(\frac{\pi y}{a}\right)\right] \hat{z}
\end{array}\right.
$$

where $a$ is the cavity dimension in the $x$-and $y$-directions, $c$ is the cavity dimension in the $z$-direction, $k_{0}=\pi \sqrt{1 / a^{2}+1 / c^{2}}$ is the wavenumber of the unperturbed resonance, $\epsilon_{0}$ and $\mu_{0}$ are the dielectric permittivity and permeability in the vacuum.

Different approaches in predicting the resonant frequency of a perturbed cavity's $T M_{110}$ mode are presented in [7]. In this paper, Slater's perturbational formulation [8] is preferred

$$
\frac{f_{1,2}-f_{0}}{f_{0}}=\frac{\int_{\Delta V}\left(\mu_{0}\left|H_{1,2}\right|^{2}-\epsilon_{0}\left|E_{1,2}\right|^{2}\right) d v}{\int_{V_{0}}\left(\mu_{0}\left|H_{1,2}\right|^{2}+\epsilon_{0}\left|E_{1,2}\right|^{2}\right) d v}
$$

where $\Delta V$ is the volume of the corner cut, $f_{0}$ and $V_{0}$ are the resonant frequency and volume of the unperturbed cavity, respectively. Replacing (3) in (4) and letting $r=s / a$ be the normalized corner cut dimension, the resonant frequencies are found as

$$
\frac{f_{1,2}}{f_{0}}=1+\frac{r^{2}}{1+a^{2} / c^{2}}\left[\operatorname{sinc}(2 \pi r) \pm \operatorname{sinc}^{2}(\pi r)\right]
$$

where $\operatorname{sinc}(x)=\sin (x) / x$.

Replacing (5) in (2) and using the first two terms of the Taylor series around $r=0$, we find the coupling coefficient, $k_{c}$, as

$$
k_{c} \approx \frac{2}{1+a^{2} / c^{2}} r^{2}
$$

The inverse function that can be used for design is

$$
r \approx\left[\frac{k_{c}\left(1+a^{2} / c^{2}\right)}{2}\right]^{\frac{1}{2}}
$$

For the special case of a triple mode rectangular cavity with $a=c$, the expressions simplify to

$$
k_{c} \approx r^{2} \text { or } r \approx \sqrt{k_{c}} .
$$

To check their validity, the analytical expressions are plotted together with the values obtained from CST Microwave Studio's Eigenmode Solver for $a / c=5 / 6$. The perturbed cavity's coupled modes' resonant frequencies are given in Fig. 3, whereas the resulting coupling coefficient is given in Fig. 4 as a function of $r$. Figs. 3 and 4 also display the results for the triangular and rounded corner cut cases as investigated below.

\section{B. Triangular Corner Cut}

The perturbation resulting from a triangular corner cut can be found using a similar procedure

$$
\begin{aligned}
\frac{f_{1,2}}{f_{0}} & =1+\frac{r^{2}}{2\left(1+a^{2} / c^{2}\right)}\left[\operatorname{sinc}^{2}(\pi r) \pm \operatorname{sinc}(\pi r)\right] \\
k_{c} & \approx \frac{1}{1+a^{2} / c^{2}} r^{2} \text { or } r \approx \sqrt{k_{c}\left(1+a^{2} / c^{2}\right)} .
\end{aligned}
$$




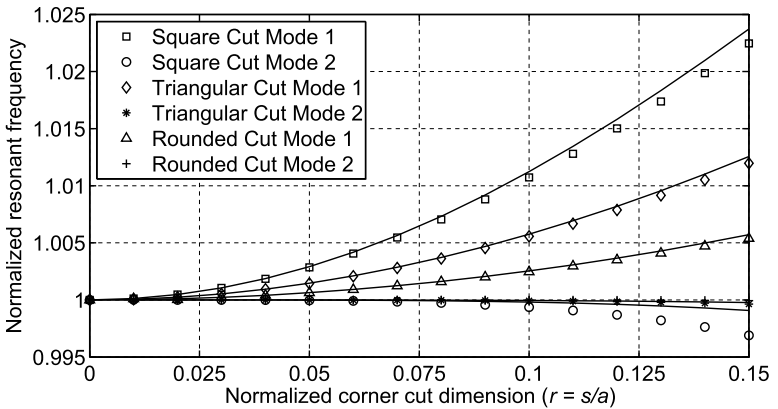

Fig. 3. Normalized resonance frequencies of the two coupled modes versus $r$ with $a / c=5 / 6$. The solid lines correspond to the results obtained analytically, whereas the symbols correspond to simulation results.

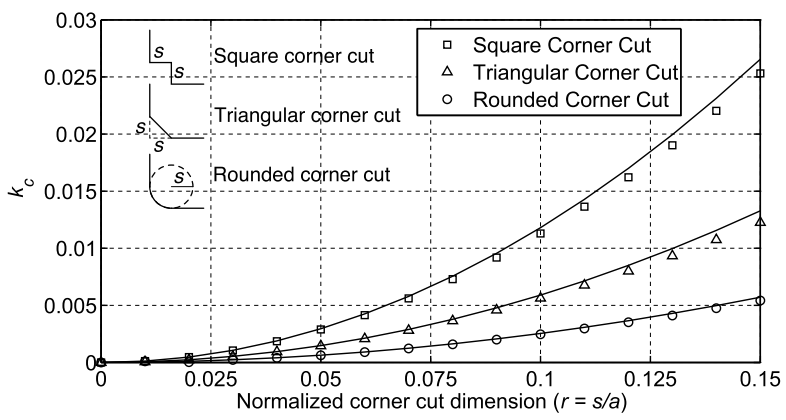

Fig. 4. Physical coupling coefficient as a function of normalized corner cut dimension with $a / c=5 / 6$. The solid lines correspond to the results obtained analytically, whereas the symbols correspond to simulation results.

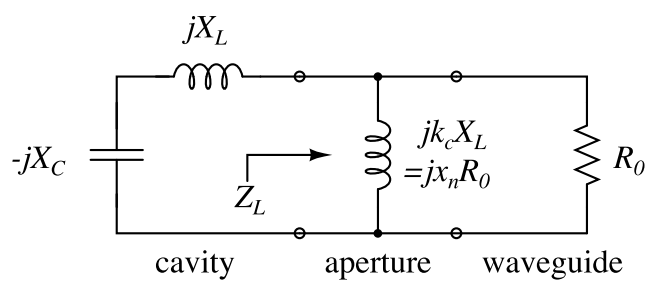

Fig. 5. Equivalent circuit for the waveguide-cavity aperture coupling structure.

\section{Rounded Corner Cut}

For a rounded corner cut, we apply the trapezoidal approximation with 64 partitions [9, p. 374] and taking up to the second-order terms, we find

$$
\begin{aligned}
& \frac{f_{1}}{f_{0}} \approx 1+0.43 \frac{r^{2}}{\left(1+a^{2} / c^{2}\right)} \text { and } \frac{f_{2}}{f_{0}} \approx 1 \\
& k_{c} \approx 0.43 \frac{r^{2}}{\left(1+a^{2} / c^{2}\right)} \text { or } r \approx\left[\frac{k_{c}\left(1+a^{2} / c^{2}\right)}{0.43}\right]^{\frac{1}{2}} .
\end{aligned}
$$

\section{WaVEguide to CAVity Coupling With A RECTANGULAR APERTURE}

The waveguide to cavity coupling structure's equivalent circuit proposed by Wheeler [10] is given in Fig. 5, where $X_{L}$ and $X_{C}$ represent the unperturbed cavity, whereas $R_{0}$ shows the load resistance presented by the waveguide. The coupling between them is represented by an inductance with $x_{n} R_{0}=k_{c} X_{L}$ [10]. Here, $x_{n}$ is the equivalent normalized shunt reactance due to an aperture placed in an infinitely long rectangular waveguide, whereas $k_{c}$ is the coupling coefficient due to an aperture placed in the common wall of two identical cavities.

For $x_{n}$, combining the results from [11]-[13] we get

$$
x_{n}=\frac{\alpha_{m} \beta_{10}}{p q}\left[1+\operatorname{sinc}\left(\frac{\pi l}{p}\right)\right]\left[\frac{2 f_{a}}{\pi f} \tan \left(\frac{\pi f}{2 f_{a}}\right)\right]
$$

where $p, q$ are waveguide cross-sectional dimensions, $\beta_{10}$ is the propagation constant of the waveguide's $T E_{10}$ mode, and $\alpha_{m}$ is the corresponding longitudinal magnetic polarizability given by [14], [15]

$$
\alpha_{m}=\frac{0.132 l^{3}}{\ln (1+0.66 l / h)} e^{-\frac{\pi t}{l}}
$$

$t$ being the thickness of the aperture. The aperture resonance frequency $f_{a}$ can be approximated by the cutoff frequency of the waveguide having the aperture's dimensions [12]: $f_{a}=$ $1 /\left(2 l\left(\epsilon_{0} \mu_{0}\right)^{1 / 2}\right)$.

On the other hand, for $k_{c}$, combining the results from [12], [13], and [15] we get

$$
k_{c}=\frac{2 \alpha_{m}}{a b c\left(1+c^{2} / a^{2}\right)}\left[1+\operatorname{sinc}\left(\frac{\pi l}{a}\right)\right]\left[\frac{2 f_{a}}{\pi f} \tan \left(\frac{\pi f}{2 f_{a}}\right)\right] \text {. }
$$

Referring to Fig. 5, the cavity loading impedance, $Z_{L}$, is given as

$$
Z_{L}=\frac{x_{n}^{2} R_{0}}{1+x_{n}^{2}}+j \frac{x_{n} R_{0}}{1+x_{n}^{2}} .
$$

Using (16) and the equivalence of the two forms of the coupling inductance, we can find the quality factor, $Q_{e}$

$$
Q_{e}=\frac{X_{L}}{\operatorname{Re}\left\{Z_{L}\right\}}=\frac{1+x_{n}^{2}}{x_{n} k_{c}} .
$$

Its value can be found using (13) and (15). The new resonant frequency of the cavity is found by including the effect of coupling aperture. Setting the imaginary part of the overall structure's equivalent impedance to zero, the new resonant frequency can be approximated as

$$
\frac{f_{r}}{f_{0}}=\sqrt{\frac{Q_{e} x_{n}}{1+Q_{e} x_{n}}}=\sqrt{\frac{1+x_{n}^{2}}{1+k_{c}+x_{n}^{2}}} \approx \sqrt{\frac{1}{1+k_{c}}} .
$$

Using the first three terms of the Taylor series for the sine and cosine functions around $\pi f /\left(2 f_{a}\right)=0$

$$
\begin{aligned}
\frac{f_{r}}{f_{0}}= & {\left[\frac{6 A^{2}\left(1+k_{c s}\right)+3}{2\left(3+k_{c s}\right)}\right.} \\
& \left.-\frac{\sqrt{36 A^{4}\left(1+k_{c s}\right)^{2}+12 A^{2}\left(k_{c s}-3\right)+9}}{2\left(3+k_{c s}\right)}\right]^{\frac{1}{2}}
\end{aligned}
$$

where $A=2 f_{a} /\left(\pi f_{0}\right)$ and $k_{c s}$ is the coupling expression provided in (15) without the Cohn's tangential frequency correction term [12].

To check the validity of the expressions, an example cubic cavity of $a=b=c=6.5 \mathrm{~mm}$ attached to a WR-28 standard waveguide ( $p=7.112 \mathrm{~mm}, q=3.556 \mathrm{~mm}$ ) via a rectangular aperture of $h=1 \mathrm{~mm}$ is analyzed for different 


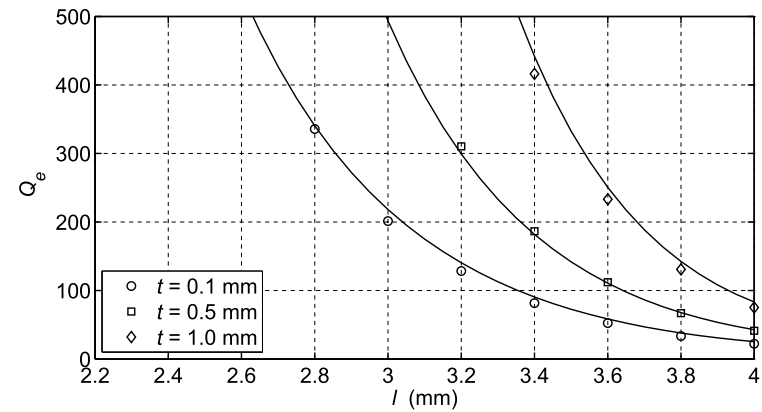

Fig. 6. $Q_{e}$ of the example aperture coupled cavity for different aperture wall thicknesses, for $h=1 \mathrm{~mm}$. The solid lines correspond to the results obtained analytically, whereas the symbols correspond to simulation results.

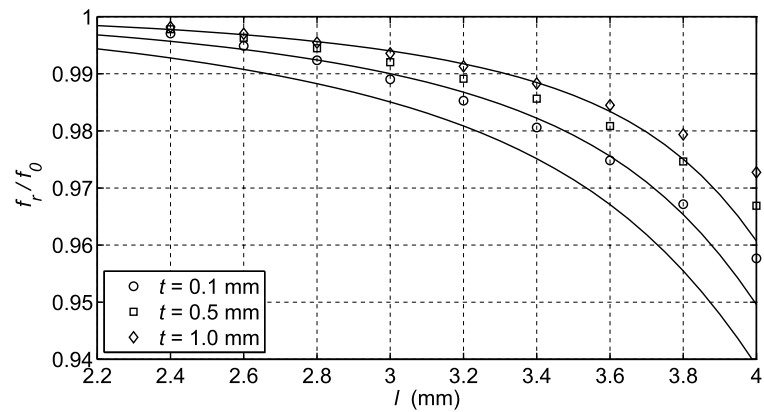

Fig. 7. Normalized resonant frequency of the example aperture coupled cavity for different aperture wall thicknesses, for $h=1 \mathrm{~mm}$. The solid lines correspond to the results obtained analytically, whereas the symbols correspond to simulation results.

values of $l$ and $t$. The structure is also simulated with CST Studio Eigenmode Solver for comparison. The results are plotted in Figs. 6 and 7. The predicted $Q_{e}$ is in good agreement with the simulation results. On the other hand, the resonant frequency prediction error increases with increasing aperture length but stays below $2 \%$ for all thickness values analyzed.

\section{INVESTIGATION OF DISPERSION}

In the ideal case, the elements of the coupling matrix are assumed frequency independent. In the previous section, we showed that $Q_{e}$ actually depends on frequency and this dependence is approximately given as

$$
Q_{e} \propto \frac{1}{\beta_{10}}\left[\frac{2 f_{a}}{\pi f} \tan \left(\frac{\pi f}{2 f_{a}}\right)\right]^{-2} .
$$

Let us define the normalized dispersion factor $\eta$ as the ratio of $Q_{e}$ normalized to its value at the center frequency $\left(f_{0}\right)$ of the filter. Then, $\eta$ is given as

$$
\eta \triangleq \frac{\left.Q_{e}\right|_{f=f_{0}}}{Q_{e}}=\eta_{w g} \eta_{a p}
$$

where $\eta_{w g}$ is due to waveguide and $\eta_{a p}$ is due to the rectangular aperture

$$
\eta_{w g}=\frac{\sqrt{f^{2}-f_{c}^{2}}}{\sqrt{f_{0}^{2}-f_{c}^{2}}} \text { and } \eta_{a p}=\left[\frac{f_{0} \tan \left(\pi / 2 \times f / f_{a}\right)}{f \tan \left(\pi / 2 \times f_{0} / f_{a}\right)}\right]^{2}
$$

where $f_{c}$ is the cutoff frequency of the waveguide. Assuming that the intermodal couplings are frequency independent
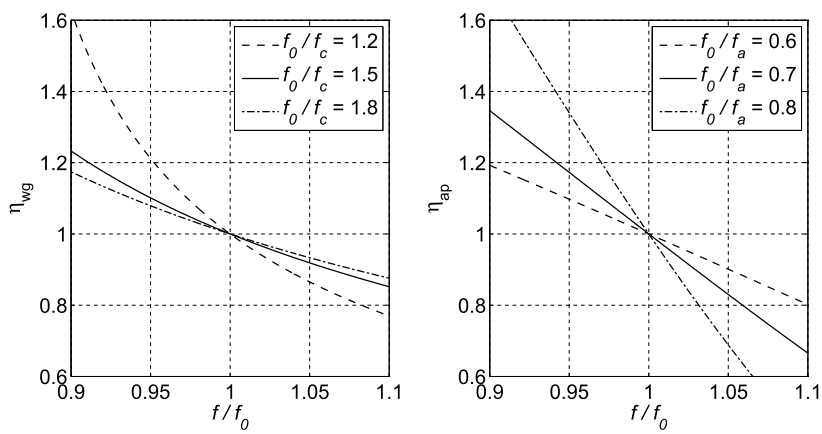

Fig. 8. Dispersion effects due to the waveguide and due to the aperture.

(valid for narrow band filters), the effect of dispersion can be implemented in the coupling matrix by multiplying the corresponding coupling element $\left(c_{S 1}\right)$ with $(21)$.

Both dispersion effects are given in Fig. 8 as a function of $f / f_{0}$ for different $f_{0} / f_{c}$ and $f_{0} / f_{a}$ ratios. It can be seen that the frequency dependence slope due to the waveguide gets higher when $f_{0}$ moves closer to $f_{c}$, hence, the distortion due to dispersion gets worse. For a specific filter, if the waveguide to be used is predefined, this effect cannot be prevented. But note that, its value is relatively small as long as $f_{0}$ is sufficiently away from the waveguide's cutoff frequency (which generally is). On the other hand, the frequency dependence slope due to the aperture gets higher when the aperture resonance frequency gets closer to $f_{0}$. The gravity of this dispersion effect can be lightened using aperture structures resulting in the same amount of coupling but having a higher resonance frequency. The search for such aperture structure is not the subject of this paper, hence rectangular-shaped irises will be used.

\section{Filter Design Algorithm}

Using the results obtained so far, the physical dimensions of a dual (triple) mode second (third)-order filter can be predicted with the following algorithm.

1) Calculate the cavity dimensions resonating at filter center frequency $f_{0}$.

2) Find the required corner cut dimension using (7).

3) Compensate for the frequency shift of the modes, as proposed in [1, p. 676], with the use of (5). The blank cavity now resonates at $f_{0}^{\prime}<f_{0}$.

4) Go to step 2 until the corner cut dimension converges.

5) Calculate the aperture length corresponding to $Q_{e}$ using (17).

6) Compensate for the frequency lowering effect of the input-output coupling irises by decreasing the length of the cavity [16, p. 463], so that the aperture coupled cavity still resonates at $f_{0}^{\prime}$ using (18).

7) Go to step 5 until the aperture and cavity length dimensions converge.

8) Recompute the required corner cut dimension using (7), based on the final values of $a$ and $c$.

\section{EXAMPLE SECOND-ORDER Filter DESIGN}

Let us design a second-order Chebyshev bandpass filter at $34 \mathrm{GHz}$, with a fractional bandwidth of $1 \%$ and an in-band 
TABLE I

PHYSICAL DIMENSIONS (IN MILLIMETERS) OF THE DESIGNED SECOND-ORDER FILTER

\begin{tabular}{|c|c|c|c|c|}
\hline$a$ & $b$ & $c$ & $s$ & $l$ \\
\hline 7.06 & 7.06 & 5.40 & 1.21 & 3.52 \\
\hline
\end{tabular}

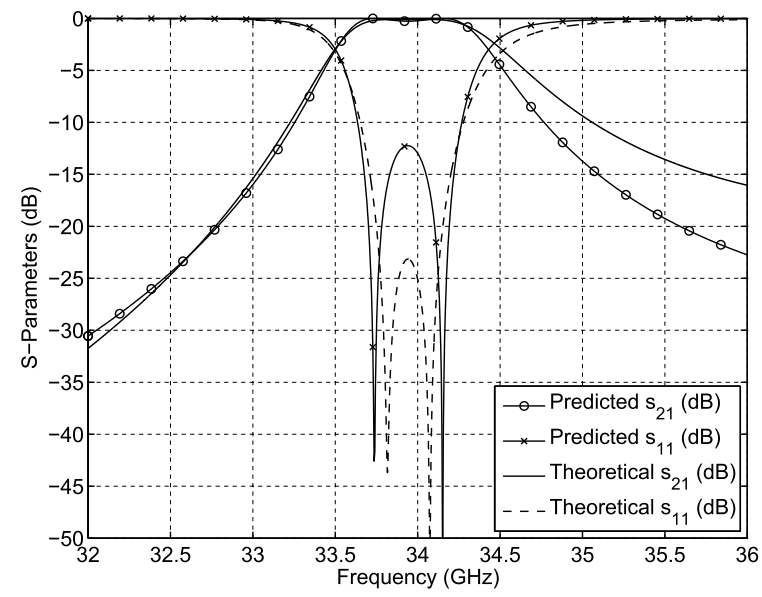

Fig. 9. Initial response of the designed filter together with the theoretical response.

return loss of $25 \mathrm{~dB}$. The corresponding normalized coupling matrix of (1) has $c_{S 1}=1.4312$ and $c_{12}=2.1670$.

The physical coupling coefficients $k_{i j}$ and $Q_{e}$ can be found from [17, pp. 516-520]

$$
k_{i j}=\frac{B}{f_{0}} M_{i j} \text { and } Q_{e}=\frac{f_{0}}{B} \frac{1}{R}
$$

where $B$ and $R$ are the bandwidth and the coupling resistance respectively. Let the input-output be WR-28 standard waveguide, the height of the coupling aperture be $1 \mathrm{~mm}$, and its thickness be $0.5 \mathrm{~mm}$. Since a dual mode cavity resonating at $f_{0}$ is not unique, let us also specify the cavity ratio $(a / c)$ as 1.25 .

Since the resonant frequencies of the resonators of a secondorder filter are located symmetrically around its center frequency, the third step of the algorithm is applied to find the length $(c)$ of the cavity that results in $\left(f_{1}+f_{2}\right) / 2=f_{0}$. Upon completion of all the steps, the dimensions given in Table I are obtained.

Fig. 9 depicts the result of CST Microwave Studio simulation run with the predicted dimensions. For this example, it can be seen that the initial response is very close to the dispersion included theoretical response without the need for optimization. In case it is not as close as desired, it is possible to do an optimization with only two parameters ( $c$ and $l$ ) assuming the intermodal coupling to be less susceptible to dimension changes.

\section{EXAMPle ThIRD-ORder Filter Design}

Let us consider a third-order filter at $34 \mathrm{GHz}$ with a $1 \%$ fractional bandwidth and an in-band return loss of $25 \mathrm{~dB}$. The normalized $5 \times 5$ coupling matrix has $c_{S 1}=1.2214$ and $c_{12}=1.2197$. Again, let the input-output be WR-28
TABLE II

PHYSiCAL DimENSIONS (IN MILLIMETERS) OF THE DESIGNED THIRD-ORDER FILTER

\begin{tabular}{|l|c|c|c|c|c|}
\hline & $a$ & $b$ & $c$ & $s$ & $l$ \\
\hline Predicted & 6.27 & 6.27 & 5.89 & 0.72 & 3.49 \\
\hline Optimized & 6.34 & 6.34 & 5.86 & 0.72 & 3.49 \\
\hline Error (\%) & 1.1 & 1.1 & 0.5 & 0 & 0 \\
\hline
\end{tabular}

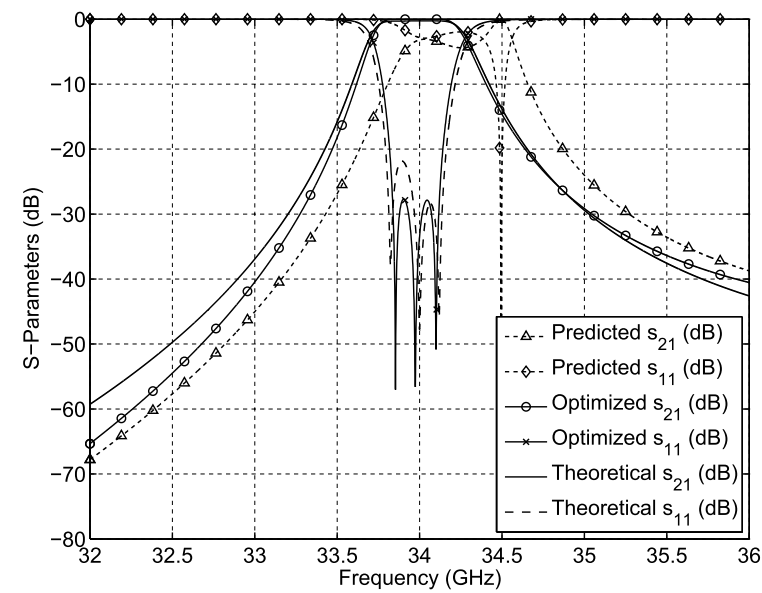

Fig. 10. S-parameters of the predicted and optimized structures together with the theoretical response.

standard waveguide, the height of the coupling aperture be $1 \mathrm{~mm}$, whereas its thickness be $0.5 \mathrm{~mm}$. This time, there is no need to specify the cavity ratio since a triple mode cavity is cubic.

On the other hand, the application of the algorithm's third and fourth steps differs from that of the second-order case. For a third-order filter, two of the resonators' resonant frequencies $\left(f_{1}\right.$ and $\left.f_{2}\right)$ are again located symmetrically around the center frequency, whereas the third resonator $\left(f_{3}\right)$ resonates at the center frequency. Therefore, the algorithm needs to be applied to find the length $(c)$ of the cavity that results in $\left(f_{1}+f_{2}\right) / 2=$ $f_{0}$, and the width $(a)$ that results in $f_{3}=f_{0}$ at the same time. Equation (5) can be used to predict all the three resonant frequencies assuming that the corner cut that couples two modes does not affect the third mode. However, note that, taking into account the directions of the corner cuts, when $a \neq c$, the dual mode approximation does not hold anymore. Therefore, while keeping the cavity cubic, the frequency compensation can be based either on $\left(f_{1}+f_{2}\right) / 2=f_{0}$ or $f_{3}=f_{0}$. Here, the first one is chosen. With $a=c$, there is no need to perform the fourth step of the algorithm, and the value $a$ that will be obtained will be a lower bound for the required value. Upon completion of all the steps, the dimensions given in Table II are obtained.

Using the initial results, an optimization is run in CST with the two cavity parameters (not three since the cavity has to be square cross sectioned) and the aperture length parameter. The corner cut dimension is left as is, assuming that the coupling coefficient will be less sensitive to dimensional changes. The obtained result is given in Fig. 10 together with the theoretical response (including the dispersion effect). 


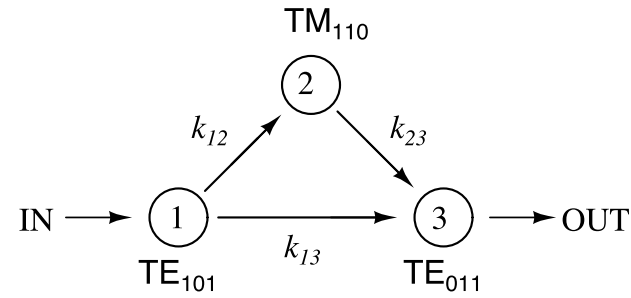

Fig. 11. Triplet coupling diagram.

Note that the obtained response rejects better at lower frequencies but worse at higher frequencies as expected in light of the results obtained in the previous sections. An interesting point is that, this dispersion caused effect is similar to that of a capacitive cross-coupling between the first and third resonators.

\section{TRiplet Structure}

One way of generating a finite frequency $\mathrm{TZ}$ in a multiple mode cavity is via the use of nonresonating node concept [18]. On the other hand, in a filter of order three, it is also possible to add the TZ by introducing a cross-coupling between the first and third resonators. The coupling diagram corresponding to this triplet structure can be seen in Fig. 11. Assuming $k_{12}$ and $k_{23}$ are positive, if the cross-coupling $\left(k_{13}\right)$ is negative, the TZ is at the lower sideband and if it is positive, the TZ is at the upper sideband [19].

In a triple mode rectangular cavity, it is possible to implement the cross-coupling with a corner cut along the input-output direction. The sign of the cross-coupling can be alternated by rotating the corner that is cut by $90^{\circ}$, since the rotation interchanges the coupled modes' resonant frequencies. Hence, third-order filters containing either a lower or an upper sideband TZ can be designed. Both cases are considered below.

\section{A. Transmission Zero at the Upper Sideband}

Consider a filter with the specifications of Section VIII and having a $\mathrm{TZ}$ at $34.75 \mathrm{GHz}$. The corresponding normalized coupling matrix is given as

$M=\begin{gathered}S \\ R_{1} \\ R_{2} \\ R_{3} \\ L\end{gathered}\left[\begin{array}{ccccc}S & R_{1} & R_{2} & R_{3} & L \\ 0 & 1.2214 & 0 & 0 & 0 \\ 1.2214 & 0.0945 & 1.1841 & 0.3455 & 0 \\ 0 & 1.1841 & -0.3052 & 1.1841 & 0 \\ 0 & 0.3455 & 1.1841 & 0.0945 & 1.2214 \\ 0 & 0 & 0 & 1.2214 & 0\end{array}\right]$.

Note that, there is an inductive (positive) coupling between the first and third resonators. This coupling is realized with a corner cut along the input-output direction. The structure can be seen in Fig. 12. The cross-coupling corner cut is divided into two parts to prevent intersection with the main coupling corner cut. The two parts are located at opposite corners so that they both result in the same type of coupling.

To determine the dimensions other than the cross-coupling corner cut dimension $\left(s_{c}\right)$, the proposed filter algorithm in

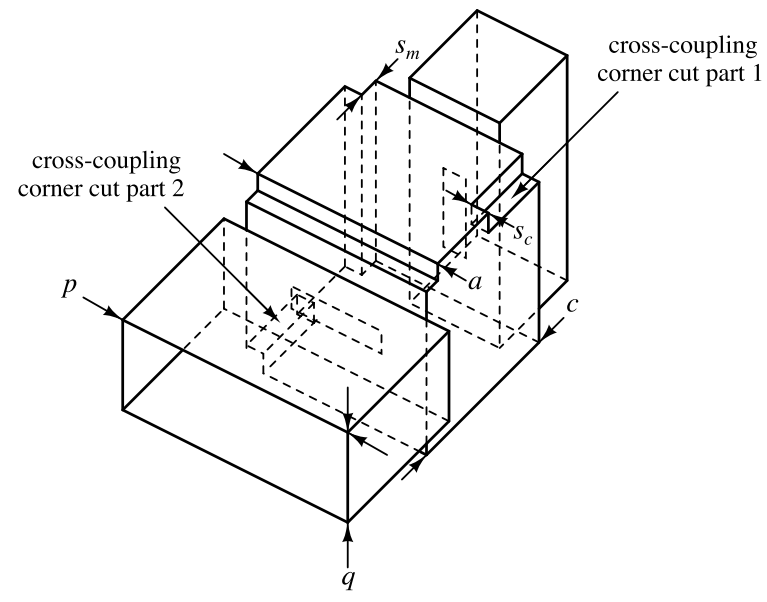

Fig. 12. Triple mode triplet structure with an upper sideband finite frequency TZ.

TABLE III

Physical Dimensions (IN Millimeters) OF THE DESIGNED TRIPLET WITH UPPER SidEBAND TZ

\begin{tabular}{|l|c|c|c|c|c|c|}
\hline & $a$ & $b$ & $c$ & $s_{m}$ & $s_{c}$ & $l$ \\
\hline Predicted & 6.27 & 6.27 & 5.88 & 0.70 & $>0.38$ & 3.49 \\
\hline Optimized & 6.33 & 6.33 & 5.89 & 0.70 & 0.40 & 3.47 \\
\hline Error (\%) & 1.0 & 1.0 & 0.2 & 0 & 5.3 & 0.6 \\
\hline
\end{tabular}

the form described in Section VIII is used. The deviations of the modes' resonant frequencies from the case with no TZ are assumed small, and are therefore neglected. On the other hand, the intermodal coupling expression in (7) is used to determine $s_{c}$. Note that the obtained value is a lower bound for the actual $s_{c}$ needed, because the generated inductive coupling needs to counteract the capacitive effect of dispersion as well. A full-wave optimization is performed based on the initial values and the final dimensions are attained. (The main coupling corner cut parameter $s_{m}$ is left out of optimization assuming that intermodal coupling is less susceptible to dimensional changes.) Both predicted and optimized values are given in Table III, whereas Fig. 13 depicts the corresponding responses together with the theoretical response.

\section{B. Transmission Zero at the Lower Sideband}

Let us move the TZ of the filter in Section IX-A to the lower sideband, at $33.25 \mathrm{GHz}$. The corresponding normalized coupling matrix is

$$
M=\begin{array}{r}
S \\
R_{1} \\
R_{2} \\
R_{3} \\
L
\end{array}\left[\begin{array}{ccccc}
S & R_{1} & R_{2} & R_{3} & L \\
0 & 1.2214 & 0 & 0 & 0 \\
1.2214 & -0.0925 & 1.1857 & -0.3377 & 0 \\
0 & 1.1857 & 0.2985 & 1.1857 & 0 \\
0 & -0.3377 & 1.1857 & -0.0925 & 1.2214 \\
0 & 0 & 0 & 1.2214 & 0
\end{array}\right] .
$$

This time, there is a capacitive (negative) coupling between the first and third resonators. This coupling is realized with a corner cut along the input-output direction rotated by 


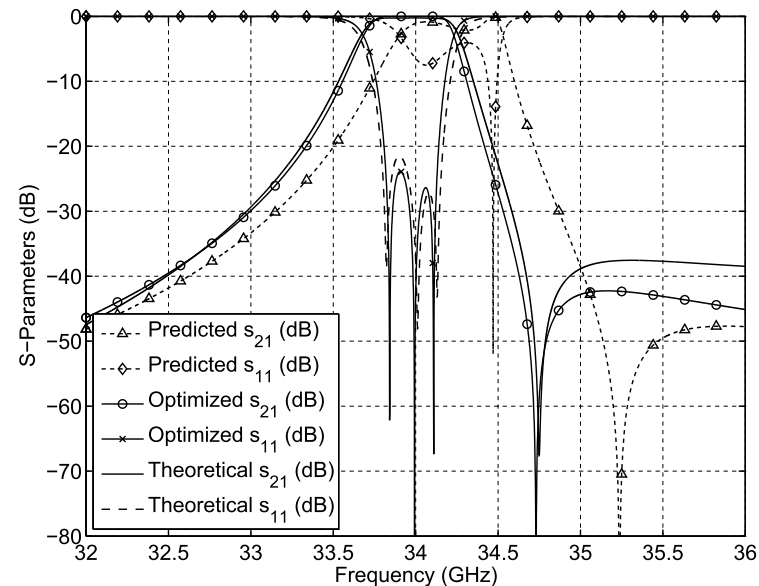

Fig. 13. Response for the triple mode triplet structure with an upper sideband finite frequency $\mathrm{TZ}$.

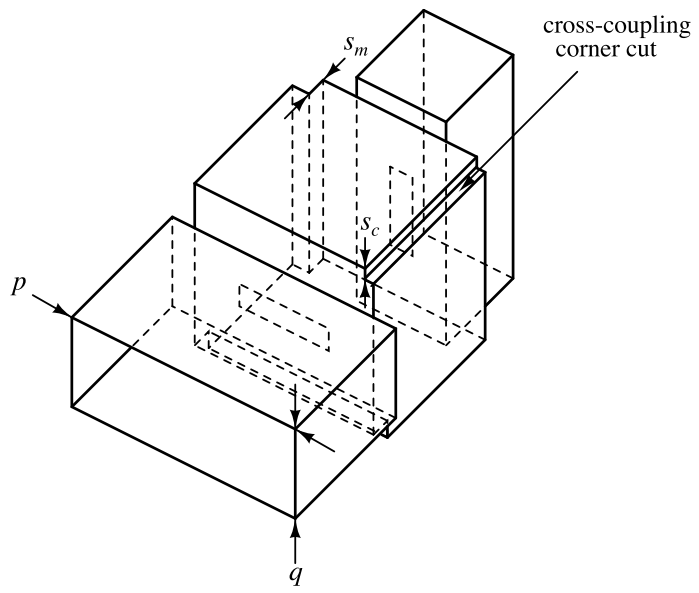

Fig. 14. Triple mode triplet structure with a lower sideband finite frequency TZ.

TABLE IV

Physical Dimensions (IN Millimeters) OF THE DESIGNED TRIPLET With LOWER SidebAND TZ

\begin{tabular}{|l|c|c|c|c|c|c|}
\hline & $a$ & $b$ & $c$ & $s_{m}$ & $s_{c}$ & $l$ \\
\hline Predicted & 6.27 & 6.27 & 5.88 & 0.71 & $<0.38$ & 3.49 \\
\hline Optimized & 6.35 & 6.35 & 5.87 & 0.71 & 0.34 & 3.47 \\
\hline Error (\%) & 1.3 & 1.3 & 0.2 & 0 & 10.5 & 0.6 \\
\hline
\end{tabular}

$90^{\circ}$ compared to the earlier design. The new structure can be seen in Fig. 14.

The dimensions are determined with the same procedure described in the previous section. Note that, because the frequency dependence of the external couplings acts like a capacitive cross coupling, the overall effective coupling becomes higher than intended. Therefore, the determined cross-coupling corner cut dimension $\left(s_{c}\right)$ is an upper bound for the actual value required. A full-wave optimization is performed based on the predicted dimensions and the final values are obtained. (The main coupling corner cut parameter $s_{m}$ is left out of optimization.) Both the predicted and optimized dimensions can be seen in Table IV, whereas the corresponding responses and the theoretical response are given in Fig. 15 for comparison.

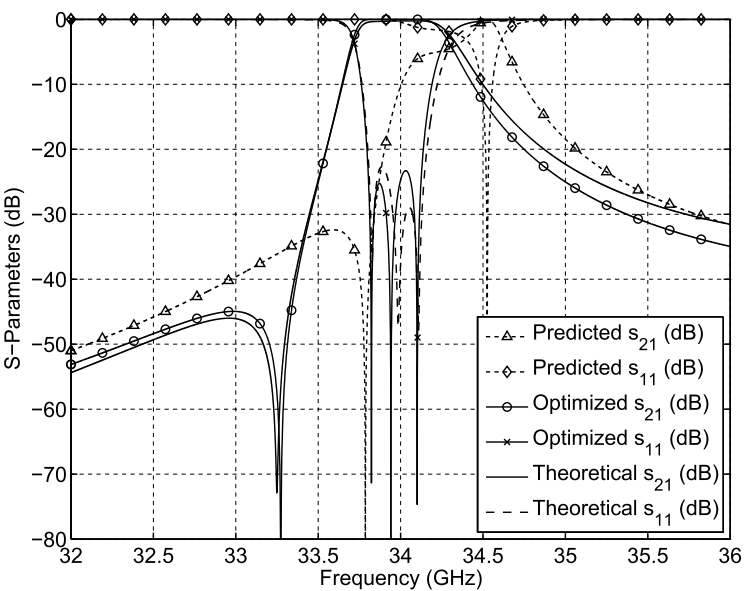

Fig. 15. Response for the triple mode triplet structure with a lower sideband finite frequency $\mathrm{TZ}$.

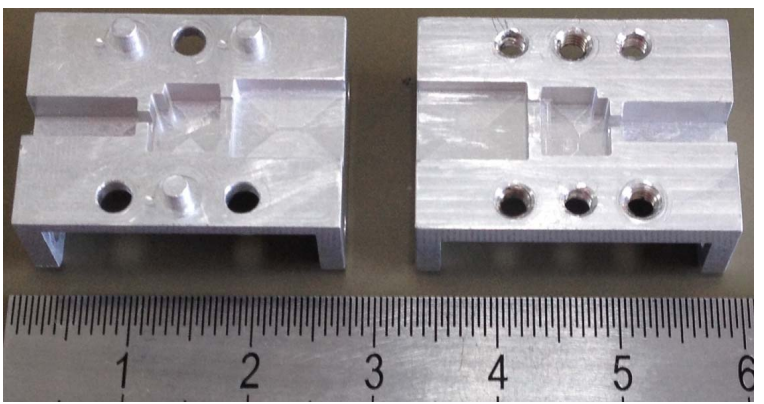

Fig. 16. Chromate conversion coated parts of the machined filter. The scale is in centimeters.

\section{EXPERIMENTAL Verification}

A $\mathrm{Ka}$-band filter with $2 \%$ fractional bandwidth, having a lower sideband TZ is designed and fabricated with CNC milling. This type of filter can be used to increase selectivity in the lower sideband, or to obtain high rejection at a specific frequency, e.g., the TZ can be adjusted to coincide with the local oscillator frequency to prevent leakage at the output of a transmitter. The chromate conversion coated parts of the fabricated filter can be seen in Fig. 16. The assembled structure weighs $10.2 \mathrm{~g}$.

Fig. 17 depicts the measurement results compared to the simulation results of the machined filter as well as the additive manufactured filter that is discussed below. The measurement results of the machined filter are in perfect agreement with the simulation results and the measured in-band insertion loss is below $0.7 \mathrm{~dB}$.

One interesting alternative manufacturing possibility is the use of novel 3-D printing technology. It is possible to print the structure's main body with the use of a polymer (wall thickness down to $0.5 \mathrm{~mm}$ attainable) and then metal coat the printed bare body. ${ }^{1}$

The same filter is also fabricated with additive manufacturing for comparison. The fabricated filter, having a minimum

\footnotetext{
${ }^{1}$ Home swissto12. [Online]. Available: http://www.swissto12.com/. Accessed: 2016-05-20.
} 


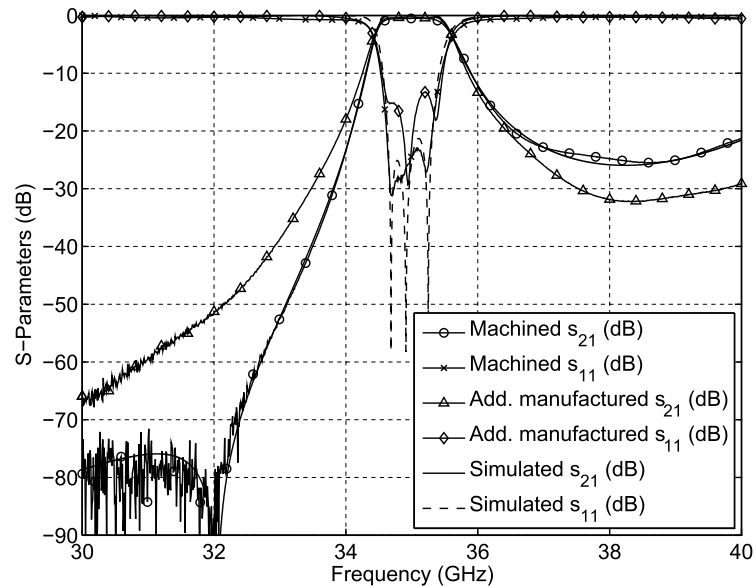

(a)

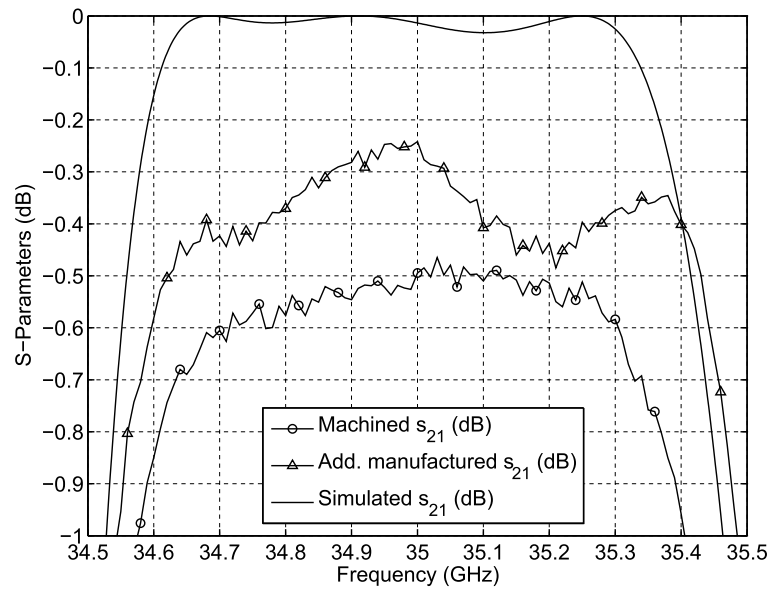

(b)

Fig. 17. Response for the fabricated triple mode triplet structure with a lower sideband finite frequency TZ. (a) S-parameters. (b) Close-up view of the insertion loss in the passband.

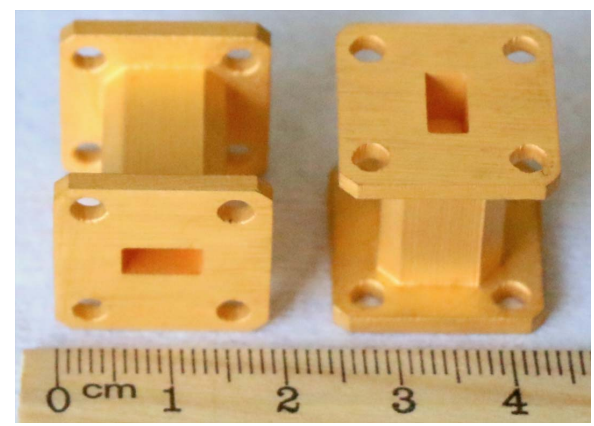

Fig. 18. Additive manufactured triple mode rectangular cavity filter.

wall thickness of $1 \mathrm{~mm}$ and $\mathrm{Cu}$ plating of $5 \mu \mathrm{m}$ with $100 \mathrm{~nm}$ Au flash on top of it, ${ }^{1}$ can be seen in Fig. 18. The input and output of the filter are designed to be used with standard WR28 waveguide flanges. The structure weighs only $4.4 \mathrm{~g}$.

The measurement results are given in Fig. 17. The in-band insertion loss is below $0.5 \mathrm{~dB}$. Note that the insertion loss of the additive manufactured filter is lower compared to the machined filter. This is an expected result since copper is a better conductor than aluminum. The usage of gold for the 3-D printed filter and chromium for the machined filter as the coating material further favors the printed filter in terms of loss. On the other hand, although the in-band responses are in agreement, the $\mathrm{TZ}$ in the response of the printed filter is missing. This is probably because the dimensions of the cross-coupling corner cut are too small to be implemented with this technology in its present state. Nonetheless, the results are very promising: additive manufacturing together with the multiple mode cavity technique is advantageous in filtering applications, especially if the weight and space have limited budgets.

\section{CONCLUSION}

In this paper, the analytical study of the multiple mode rectangular cavity is pursued. Expressions are obtained for the resonant frequency shift and external $Q$ of the aperture coupled cavity, and for the intermodal coupling due to various corner cut shapes.

An analysis is performed on the dispersion characteristic of the rectangular aperture coupled multiple mode cavity filter. Upon implementing the expression obtained in the coupling matrix of a given filter, the dispersed response can be predicted.

An algorithm is used to predict the physical dimensions of the multiple mode cavity filter. Example dual mode secondorder and triple mode third-order filters are designed using the algorithm provided.

With the addition of an extra corner cut, the triple mode rectangular cavity structure is altered to form a triplet structure. Filters having TZs at the upper and lower sidebands are designed.

An example design is fabricated with both machining and additive manufacturing. The additive manufactured $\mathrm{Ka}$-band third-order triple mode cavity filter structure weighs only $4.4 \mathrm{~g}$ and the experimental results are in agreement with the expectations.

\section{REFERENCES}

[1] G. L. Ragan, Microwave Transmission Circuits (MIT, Radiat. Lab. Series), vol. 9. New York, NY, USA: McGraw-Hill, 1948.

[2] W.-G. Lin, "Microwave filters employing a single cavity excited in more than one mode," J. Appl. Phys., vol. 22, pp. 989-1001, Aug. 1951.

[3] X.-X. Liang, K. A. Zaki, and A. E. Atia, "Dual mode coupling by square corner cut in resonators and filters," IEEE Trans. Microw. Theory Techn., vol. 40, no. MTT-12, pp. 2294-2302, Dec. 1992.

[4] G. Lastoria, G. Gerini, M. Guglielmi, and F. Emma, "CAD of triplemode cavities in rectangular waveguide," IEEE Microw. Guided Wave Lett., vol. 8, no. 10, pp. 339-341, Oct. 1998.

[5] S. Amari, "Application of representation theory to dual-mode microwave bandpass filters," IEEE Trans. Microw. Theory Techn., vol. 57, no. 2, pp. 430-441, Feb. 2009.

[6] R. F. Harrington, Time-Harmonic Electromagnetic Fields (IEEE Press Series Electromagn. Wave Theory). Hoboken, NJ, USA: Wiley, 2001.

[7] S. R. Nelatury and C. F. Nelatury, "A comparison of perturbation formulas for a square electromagnetic resonator," IEEE Antennas Propag. Mag., vol. 56, no. 1, pp. 130-142, Feb. 2014

[8] D. M. Pozar, Microwave Engineering. Hoboken, NJ, USA: Wiley, 2011.

[9] G. B. Thomas, Jr., R. L. Finney, M. D. Weir, and F. R. Giordano, Thomas' Calculus. Reading, MA, USA: Addison-Wesley, 2003. 
[10] H. A. Wheeler, "Coupling holes between resonant cavities or waveguides evaluated in terms of volume ratios," IEEE Trans. Microw. Theory Techn., vol. MTT-12, no. 2, pp. 231-244, Mar. 1964.

[11] R. E. Collin, Field Theory of Guided Waves (IEEE AP-S: The IEEE/OUP Series Electromagn. Wave Theory). Piscataway, NJ, USA: IEEE Press, 1991.

[12] S. B. Cohn, "Microwave coupling by large apertures," Proc. IRE, vol. 40, no. 6, pp. 696-699, Jun. 1952.

[13] R. Levy, "Improved large aperture waveguide coupling theory," in IEEE MTT-S Int. Microw. Symp. Dig., Apr./May 1979, pp. 431-433.

[14] N. A. McDonald, "Simple approximations for the longitudinal magnetic polarizabilities of some small apertures," IEEE Trans. Microw. Theory Techn., vol. 36, no. 7, pp. 1141-1144, Jul. 1988.

[15] N. A. McDonald, "Electric and magnetic coupling through small apertures in shield walls of any thickness," IEEE Trans. Microw. Theory Techn., vol. MTT-20, no. 10, pp. 689-695, Oct. 1972.

[16] G. L. Matthaei, E. M. T. Jones, and L. Young, Microwave Filters, Impedance-matching Networks, and Coupling Structures. Norwood, MA, USA: Artech House, 1980.

[17] R. J. Cameron, R. Mansour, and C. M. Kudsia, Microwave Filters for Communication Systems: Fundamentals, Design and Applications. Hoboken, NJ, USA: Wiley, 2007.

[18] S. Amari, U. Rosenberg, and J. Bornemann, "A novel approach to dual and triple-mode pseudo-elliptic filter design," in Proc. 34th Eur. Microw. Conf., vol. 2. Oct. 2004, pp. 993-996.

[19] J. B. Thomas, "Cross-coupling in coaxial cavity filters-A tutorial overview," IEEE Trans. Microw. Theory Techn., vol. 51, no. 4, pp. 1368-1376, Apr. 2003.

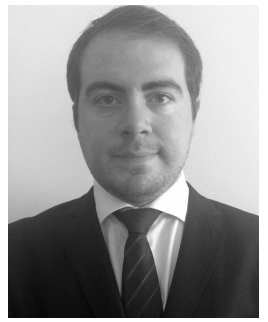

Ceyhun Kelleci (S'12) received the B.S. and M.S. degrees from the Electrical and Electronics Engineering Department, Bilkent University, Ankara, Turkey, in 2008 and 2011, respectively, where he is currently pursuing the Ph.D. degree.

Since 2011, he has been with Meteksan Defence Ind. Inc., Ankara, Turkey, as a Microwave Design Engineer. His current research interests include microwave cavity filters and power amplifiers.

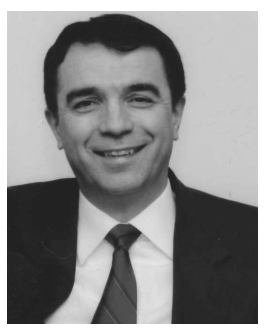

Abdullah Atalar (F'10) received the B.S. degree from Middle East Technical University, Ankara, Turkey, in 1974, and the M.S. and Ph.D. degrees from Stanford University, Stanford, CA, USA, in 1976 and 1978, respectively, all in electrical engineering.

He was with Hewlett-Packard Labs, Palo Alto, CA, USA, in 1979. From 1980 to 1986, he was on the faculty of the Middle East Technical University. In 1986, he joined the Bilkent University, Ankara, as the Chairman of the Electrical and Electronics Engineering Department and served on the founding of the department where he is currently a Professor. In 1995, he was a Visiting Professor with Stanford University. From 1996 to 2010, he was the Provost of Bilkent University, where he is currently the Rector. His current research interests include micromachined devices and microwave electronics.

Dr. Atalar was the recipient of the Science Award of TUBITAK in 1994. 